\title{
Percepción de riesgo y factores asociados al consumo de drogas legales e ilegales en estudiantes de la Universidad de Boyacá1
}

\author{
Pérez Prada, Mónica Patricia²; Barreto Bedoya, Paola 3; Roa Cubaque, Marcela \\ América4; Rubiano Díaz, Guiomar Haydees.
}

\begin{abstract}
Resumen |El objetivo de este artículo es describir la percepción de riesgo y los factores de protección y de riesgo frente al consumo de sustancias psicoactivas legales e ilegales en estudiantes de la Universidad de Boyacá. Es un estudio cuantitativo y descriptivo de corte transversal, para el cual se tomó una muestra de 573 estudiantes que fueron seleccionados mediante muestreo estratificado probabilístico. El instrumento utilizado fue la encuesta sobre consumo de drogas, factores y percepción de riesgo en estudiantes universitarios (CODEU) diseñado por Beverido, Salas, De San Jorge, Pavón, Blázquez, Gogeascoechea y Cruz (2012),
\end{abstract} Editado por William Alejandro Jiménez-Jiménez Universidad de Boyacá, Colombia.

\section{Revisado por}

William Alejandro Jiménez-Jiménez Universidad de Boyacá

\author{
${ }^{2}$ Monica Patricia Pérez Prada \\ [ $\triangle]$ \\ 3 Paola Barreto Bedoya \\ ${ }_{4}$ Marcela América Roa \\ Cubaque \\ 5 Guiomar Haydee Rubiano \\ Díaz
}

Coordinadora Proyección Social Facultad de Ciencias Humanas y Educativas. Integrante del grupo de investigación ETHOS. Contacto: mpperez@ uniboyaca.edu.co

Jefe Departamento de Salud Pública. Integrante del grupo de Investigación HYGEA. Contacto: paolabarreto@uniboyaca.edu.co

Jefe Departamento de Ciencias Básicas. Integrante del grupo de investigación OXIGENAR. Contacto: maroa@uniboyaca.edu.co

Directora Programa de Terapia Respiratoria. Integrante del Grupo de Investigación OXIGENAR. Contacto: ghrubiano@uniboyaca.edu.co

\footnotetext{
${ }^{1}$ Este artículo se deriva del proyecto de investigación titulado: Diagnóstico de Percepción, Riesgo y Consumo de Drogas en Estudiantes de la Universidad de Boyacá: Evidencias para el diseño de estrategias para la Prevención.
} 
que consta de 73 preguntas. Este instrumento fue validado y obtuvo un alpha de Cronbach de 0.787 . Los hallazgos muestran que entre los factores protectores se encuentran: adecuadas relaciones familiares y practicar un deporte, como factores de riesgo se identifican principalmente: la permisividad social y las construcciones de reconocimiento social presentes ante el consumo de sustancias legales. De las sustancias ilegales, la marihuana es la que se percibe como de menor riesgo frente a la cocaína, el bazuco, los inhalantes y la heroína.

Palabras clave | Sustancias Psicoactivas, factores de protección, factores de riesgo, percepción de riesgo.

Abstract | The aim of this article is to describe the perception of risk, protective factors and risk of using legal and illegal psychoactive substances in students from the "Universidad de Boyacá". This is a quantitative, cross-sectional descriptive study, for which a sample of 573 students, who were selected through stratified probability sample, was taken. The instrument used was a survey on drug use, risk factors and outcomes in university students (CODEU, 2012) designed by Beverido, Salas, De San Jorge, Pavón, Blázquez, Gogeascoechea and Cruz (2012), consisting of 73, which was validated and obtained a Cronbach's alpha of 0.787 . The findings show that among protective factors we find adequate family relationships and practicing a sport. As risk factors social permissiveness and social recognition constructions present in the use of legal substances are mainly identified. From the illegal substances, marijuana is perceived as the one having the less risk compared to cocaine, crack cocaine, inhalants and heroin.

Keywords | Psychoactive Substances, protective factors, risk factors, risk perception.

\section{Introducción}

El consumo de sustancias psicoactivas (SPA) es un fenómeno que ha acompañado a los seres humanos desde hace aproximadamente 50.000 años (Winkelman, 2006). Estas se han relacionado con la alteración de los estados de conciencia y han tenido diferentes usos: medicinales 
o terapéuticos, como generadores de emociones y estados del ánimo, como elementos de fuga de la realidad o generadores de ideas, como mecanismos nutricionales, medios de inspiración, y en algunos casos como un mediador en la comunicación con deidades. Sin embargo, en la actualidad el uso de estas sustancias es considerado un problema de salud pública por las consecuencias asociadas a su consumo. Se afirma que fenómenos como la globalización, el narcotráfico y los medios de comunicación, han impuesto una nueva manera de consumo caracterizado por su falta de sentido y de significado, lo que ha dimensionado métodos de consumo cada vez más poderosos y peligrosos para la salud física y mental (Ministerio de la Protección Social e Instituto Colombiano de Bienestar Familiar, 2010).

La Política Nacional para la reducción del consumo de Sustancias Psicoactivas, las ha definido como toda sustancia de origen natural o sintético, lícita o ilícita, que se introduce al organismo (sin prescripción médica) con la intención de alterar la percepción, la conciencia o cualquier otro estado psicológico (Ministerio de la Protección Social, 2007). Entre ellas se pueden encontrar: Tabaco, bebidas alcohólicas, marihuana, cocaína, alucinógenos, inhalantes, metanfetaminas, bebidas energizantes, tranquilizantes, anfetaminas y estimulantes.

El consumo de Sustancias Psicoactivas ha sido ampliamente estudiado desde diferentes áreas de desarrollo del ser humano como: personal, familiar y social. Uno de los temas de interés frente a esta problemática ha sido identificar los factores de protección y de riesgo asociados, los cuales han sido definidos por la literatura científica como "una serie de circunstancias o eventos de naturaleza biológica, psicológica, social, o ambiental cuya presencia o ausencia modifican la probabilidad de la aparición de un problema" (Morales, Plazas, Sánchez \& Arena; 2011, p. 675).

De esta manera, se han identificado factores ambientales que se relacionan con la disponibilidad y el acceso a una sustancia. Factores individuales como motivaciones, curiosidad, evasión de situaciones problemáticas, necesidad de aceptación de grupo, hedonismo y rebeldía; así como carencia de objetivos, ilusiones y aspiraciones. Factores genéticos que están relacionados con patrones de consumo presentes en algún miembro de la familia. Factores psicosociales relacionados con características del entorno social, con características de personalidad y con la salud mental (Cid-Monckton \& Pedrao, 2011). 
Asimismo han sido clasificados como de riesgo o de protección y se definen de la siguiente manera: Los factores de riesgo son todas aquellas situaciones sociales, personales o interpersonales que están presentes en los contextos donde se desarrollan los individuos y que pueden llegar a incrementar la probabilidad de consumo de sustancias psicoactivas o la adicción a una de ellas (Rivolta, 2012). De la misma manera, hacen alusión a las características y variables que elevan la probabilidad de desarrollar una patología en relación con la adicción (Arias, Calderón, Cano \& Castaño, 2012). En general, se puede afirmar que un factor de riesgo para el consumo de SPA hace referencia a todos aquellos eventos, condiciones, sucesos, situaciones, comportamientos de tipo biológico, psicológico o social que aumentan la probabilidad de consumo o adicción. Por otro lado, los factores de protección son los que pueden reducir, contrarrestar o eliminar la probabilidad de existencia de un proceso adictivo; sin embargo, se afirma que ninguno de los factores, sea de riesgo o de protección, pueden predecir las conductas de consumo y solo se puede hablar en términos probabilísticos (Rivolta, 2012).

En Tunja, Manrique, Ospina y García (2009), realizaron un estudio cuyo objetivo era caracterizar el consumo de tabaco y alcohol en adolescentes escolarizados entre 13 y 18 años de este municipio, al igual que identificar los factores psicosociales de riesgo. Entre sus principales hallazgos se encuentra que el alcohol es la sustancia que registra mayor prevalencia de consumo con una tasa de $96.5 \%$ para las mujeres y un $75.8 \%$ para los hombres, también se identificó que la edad promedio de inicio para el consumo de Sustancias Psicoactivas ha disminuido a los 12 años en los dos géneros. Como factores de riesgo se encuentran: la maduración temprana, antecedentes familiares de desórdenes conductuales, de dependencia de alcohol y drogas lícitas, poca tolerancia a la frustración, problemas y disfunción familiar. Entre los factores desencadenantes escolares se identifican el mal rendimiento académico, problemas con los profesores o los compañeros y pérdida de años. De la misma manera pertenecer a una comunidad violenta y con disponibilidad de drogas, al igual que la facilidad para el acceso a bebidas alcohólicas y cigarrillo, se constituyen como factores de riesgo.

En esta misma línea, Peñaloza, Pérez, Rincón y Manrique (2010), caracterizaron el comportamiento del consumo de Sustancias Psicoactivas y los factores psicosociales determinantes para estudiantes universitarios mayores de edad de Tunja. Se encontró que $94.6 \%$ de los encuestados han consumido alcohol en algún momento de su vida, un $71.4 \%$ para el consumo 
de tabaco, $18.7 \%$ para marihuana y $34.1 \%$ para energizantes. En lo relacionado con el género, el masculino presenta mayor porcentaje de consumo de alcohol con el $95.9 \%$ con respecto del femenino con 93.4\%. La edad media de los participantes fue de 22.13 años.

Otro estudio identificado en el ámbito nacional fue el desarrollado por Cáceres, Salazar, Varela y Tovar (2006) en la ciudad de Cali, quienes tenían como objetivo identificar el consumo de drogas legales e ilegales y los factores de riesgo y protección psicosociales asociados al consumo. Se encontró que la droga legal más consumida es el alcohol y la ilegal es la marihuana. Los resultados revelan que el consumo de SPA legales e ilegales tiene un importante componente social, como el uso de estas sustancias en relación con actividades de ocio, diversión y eventos sociales, en las cuales otras personas han participado, lo que provoca el comportamiento de consumo. Además se identificó que la baja impulsividad, una mayor tolerancia a la frustración, una mejor aceptación de la norma y mayor autorregulación, se constituyen como factores protectores. Por el contrario, indicadores de trastornos de conducta, de déficit de atención con hiperactividad, la conducta antisocial, el comportamiento desafiante y el maltrato, parecen constituir elementos de riesgo en los jóvenes. El estudio concluye que la ausencia o presencia de personas consumidoras es el principal factor que actúa como de riesgo o de protección frente al consumo de todas las sustancias, tanto legales como ilegales.

En México se encontró que los factores de riesgo correlacionados con el uso de Sustancias Psicoactivas están relacionados con el consumo de drogas en la familia, un débil apoyo y control familiar, el abandono o suspensión de estudios y una baja adherencia escolar, la tolerancia social y la disponibilidad de sustancias, la pertenencia a redes sociales disfuncionales y un uso inadecuado del tiempo libre, así como diversos trastornos de conducta, afectivos y psicológicos, entre los que se cuentan la depresión y la ansiedad (Arellanez, Díaz, Wagner \& Pérez, 2004).

Es importante nombrar que la percepción de riesgo ha estado presente en muchos estudios relacionados con el uso y abuso de SPA y se ha considerado como una de las variables de mayor impacto en la iniciación y mantenimiento del consumo (García del Castillo, 2012). La percepción se trata de un proceso cognitivo que depende de la información de cada persona acerca de diferentes contextos, objetos y otras personas, la cual se procesa de forma inmediata originando un juicio o una postura, que puede condicionar el comportamiento (Pastor, 2000). 
La historia personal, la cantidad y calidad de la información, las creencias y las actitudes, los estereotipos y la motivación influyen en la percepción del riesgo (García del Castillo, 2012), y esta a su vez influirá en la conducta de consumo de SPA.

Lo expuesto con anterioridad evidencia la necesidad de desarrollar estudios que permitan explicar el fenómeno del consumo de SPA y los factores de riesgo y de protección asociados en diferentes grupos poblacionales, con el fin de proponer y desarrollar estrategias que mitiguen la problemática y sus efectos, pues como ya se mencionó anteriormente, es considerada como un problema de salud pública que afecta directamente la salud mental del individuo, la familia y la comunidad.

\section{Método}

\section{Tipo de investigación}

La investigación se realizó con un enfoque cuantitativo porque utilizó la recolección y análisis de datos para contestar preguntas de investigación establecidas previamente; de tipo descriptivo ya que mide y evalúa diversos aspectos, dimensiones o componentes del fenómeno investigado; de corte transversal debido a que la toma de datos se efectuó en un período determinado de tiempo (Hernández, Fernández \& Baptista, 2010).

\section{Población}

5205 estudiantes de la Universidad de Boyacá matriculados en pregrado durante el primer semestre académico de 2015 de las sedes de Chiquinquirá, Sogamoso y Tunja, de acuerdo con la información proporcionada por el Sistema Integrado de Información de la Universidad de Boyacá.

\section{Muestra}

La muestra estuvo conformada por 573 estudiantes de la Universidad de Boyacá, los cuales se seleccionaron mediante muestreo probabilístico estratificado (Hernández, Fernández \& 
Baptista, 2010), se realizó inicialmente una estratificación por sede, posteriormente por facultad y finalmente por programa; como criterios de inclusión se tuvo en cuenta que fueran estudiantes de pregrado de la universidad de Boyacá y manifestar su voluntad de participar en la investigación por medio del diligenciamiento del consentimiento informado. Se excluyeron los estudiantes con diagnóstico de trastornos neurológicos y menores de edad.

\section{Instrumentos}

La información se recolectó con el instrumento denominado Encuesta sobre consumo de drogas, factores y percepción de riesgo en estudiantes universitarios (CODEU) diseñado por Beverido, Salas, De San Jorge, Pavón, Blázquez, Gogeascoechea y Cruz (2012), a partir de la adaptación autorizada de Villatoro et al. (2009), el cual consta de 73 preguntas que indagan sobre aspectos sociodemográficos, consumo de drogas licitas e ilícitas, factores de protección y de riesgo, percepción de riesgo y sobre las campañas de prevención emprendidas por diferentes instituciones.

El instrumento fue piloteado y validado estadísticamente obteniendo un índice de confiabilidad de 0.787 utilizando el Alpha de Cronbach, lo que permite señalar que se trata de un instrumento confiable.

\section{Definición de Variables}

Factores de riesgo: Son todas aquellas situaciones sociales, personales o interpersonales que están presentes en los contextos donde se desarrollan los individuos y que pueden llegar a incrementar la probabilidad de consumo de Sustancias Psicoactivas o la adicción a una de ellas (Rivolta, 2012).

Factores de protección: hace referencia a todas aquellas situaciones, condiciones o eventos de tipo biológico, psicológico, social o cultural que pueden reducir, contrarrestar o eliminar la probabilidad de existencia de un proceso adictivo (Rivolta, 2012). 


\section{Procedimiento}

Se contactó a las directivas de la Universidad de Boyacá con el fin de solicitar su autorización para el desarrollo de la investigación. Posteriormente se escogieron al azar el número de estudiantes establecido en el muestreo, a quienes se les entregó el consentimiento informado para ser diligenciado. Se dio a conocer a los estudiantes el objetivo de la investigación y la forma en que se usaría la información suministrada, asimismo se explicó el diligenciamiento de la prueba de acuerdo con los patrones establecidos en la misma.

\section{Resultados}

Se mostrarán inicialmente los resultados relacionados con los factores de riesgo y de protección identificados para esta problemática y posteriormente los de percepción de riesgo.

\section{Factores de riego y de protección}

En este estudio se contemplaron principalmente como factores de riesgo y/o de protección: el uso del tiempo libre, la influencia de pares, factores familiares, auto concepto y autoestima.

Uso del tiempo libre

Tabla 1 / Distribución de la población de acuerdo con el Uso del tiempo libre

\begin{tabular}{lccc}
\hline \hline \multicolumn{1}{c}{ ACTIVIDAD } & NUNCA & $\begin{array}{c}\text { ALGUNAS } \\
\text { VECES }\end{array}$ & $\begin{array}{c}\text { CASI } \\
\text { SIEMPRE }\end{array}$ \\
\hline \hline Practico algún deporte & $17 \%$ & $61 \%$ & $22 \%$ \\
Me reúno con la banda/pandilla de mi barrio & $88 \%$ & $10 \%$ & $2 \%$ \\
Ayudo en las labores de la casa & $7 \%$ & $43 \%$ & $50 \%$ \\
No hago nada en especial & $39 \%$ & $48 \%$ & $13 \%$ \\
Hago tarea o estudio & $4 \%$ & $33 \%$ & $63 \%$ \\
Paso el tiempo jugando videojuegos o viendo TV & $25 \%$ & $58 \%$ & $17 \%$ \\
Salgo a pasear con mi familia & $13 \%$ & $67 \%$ & $21 \%$ \\
Me voy a beber con mis amigos/as & $36 \%$ & $59 \%$ & $6 \%$ \\
Asisto a clases artísticas & $62 \%$ & $28 \%$ & $9 \%$ \\
Me aburro la mayor parte del tiempo & $49 \%$ & $43 \%$ & $8 \%$ \\
\hline
\end{tabular}

$\boldsymbol{\epsilon} \| 90$ 
Frente al uso del tiempo libre, la tabla 1 muestra que en la frecuencia casi siempre, la mayoría de los estudiantes lo utiliza para hacer tareas o estudiar (63\%) y en segundo lugar para ayudar con las labores de la casa (50\%). Para la categoría algunas veces, las actividades que desarroIlan son: Practico algún deporte (61\%), salgo con mi familia (67\%), voy a beber con amigos (59\%), juego videojuegos o veo T.V (58\%), y el $48 \%$ no hace nada especial.

Me voy a beber con amigos es otra de las situaciones contempladas, es una conducta de consumo entendida como factor de riesgo para el consumo de cualquier SPA ilegal y legal. En la categoría nunca, el $62 \%$ de los participantes afirma no asistir a clases de artísticas, se aburre (49\%) y el $88 \%$ afirma que nunca se reúne con bandas o pandillas del barrio, situación que puede favorecer las conductas de no consumo de SPA.

Influencia de pares

Tabla 2 / Distribución de la población de acuerdo con la Influencia de pares

\begin{tabular}{lcccc}
\hline \hline & NINGUNO & ALGUNOS & LA MAYORÍA & TODOS \\
\hline \hline Estudian en una Universidad & $4 \%$ & $40 \%$ & $40 \%$ & $16 \%$ \\
Fuman cigarros & $19 \%$ & $57 \%$ & $21 \%$ & $3 \%$ \\
Practican algún deporte & $11 \%$ & $57 \%$ & $28 \%$ & $4 \%$ \\
Tienen buenas calificaciones en la & $2 \%$ & $50 \%$ & $45 \%$ & $3 \%$ \\
universidad & $37 \%$ & $57 \%$ & $6 \%$ & $0 \%$ \\
Tienen problemas con sus padres & $6 \%$ & $42 \%$ & $39 \%$ & $13 \%$ \\
Consumen bebidas alcohólicas & $61 \%$ & $35 \%$ & $4 \%$ & $0 \%$ \\
Consumen drogas & & &
\end{tabular}

En cuanto a la influencia de los pares, se encontró que el $56 \%$ de los amigos de los estudiantes encuestados pertenece a la universidad. En cuanto al porcentaje de los amigos que fuman se resalta el porcentaje de algunos con $57 \%$ y la mayoría con $21 \%$. Por otro lado, el $32 \%$ de los participantes respondió que la mayoría de sus amigos practica algún deporte y el $48 \%$ tiene buenas calificaciones; sin embargo, se identifica un porcentaje importante frente a la frecuencia algunos. 
En lo relacionado con los conflictos familiares de los amigos, se resalta como aspecto positivo que solo el $6 \%$ de los participantes reporta la categoría la mayoría, el porcentaje restante muestra solo algunos y ninguno. Al preguntar a los participantes si sus amigos consumen bebidas alcohólicas, el $42 \%$ reporta que la mayoría y todos usan este tipo de sustancias. Frente al uso de drogas, el $61 \%$ de amigos de los estudiantes que participaron en el estudio no consume drogas.

Con respecto a la presión ejercida por los amigos para consumir alcohol, el estudio identificó que el $40 \%$ de los participantes no ha sido presionado para emborracharse, el $31.4 \%$ un poco, el $21.5 \%$ se ha sentido algo presionado y el 7.2 muy presionado. Al indagar por la presión ejercida por los amigos para consumir drogas se identificó que tan solo el $1.6 \%$ de los estudiantes ha sido presionado para consumir SPA distintos al alcohol, contrario a esto, el $84.3 \%$ no ha sido presionado, seguramente por la valoración negativa construida frente a las sustancias ilegales.

Factores familiares

Tabla 3 / Distribución de la población de acuerdo con el factor familiar, relación con padres o sustitutos

\begin{tabular}{lcccc}
\hline \hline & EXCELENTE & BUENA & REGULAR & MALA \\
\hline \hline $\begin{array}{l}\text { ¿Cómo considera la relación con su (s) } \\
\text { padres o sustitutos? }\end{array}$ & $48.5 \%$ & $41.2 \%$ & $9.6 \%$ & $0.7 \%$ \\
\hline
\end{tabular}

En cuanto a los factores familiares, la tabla 3 muestra que los participantes consideran que la relación con sus padres es excelente en el $48.5 \%$, buena en el $41.2 \%$; esto corresponde al $89.7 \%$ de la muestra, lo que permite afirmar que este el factor de protección más frecuente en los participantes. De igual manera se enfatiza la importancia de estas relaciones ya que, como se ha mencionado anteriormente, están directamente relacionadas con los patrones de consumo. 
Tabla 4 / Distribución de la población de acuerdo con el consumo de alcohol por parte de los padres después de peleas

\begin{tabular}{lccc}
\hline \hline & sí & NO & NO SE PELEAN \\
\hline \hline $\begin{array}{l}\text { Cuándo se han peleado sus padres calguno de ellos } \\
\text { ha bebido alcohol? }\end{array}$ & $18 \%$ & $62 \%$ & $20 \%$ \\
\hline
\end{tabular}

El consumo de alcohol relacionado con conflictos familiares no es una situación frecuente en este grupo poblacional, ya que tan solo el $18 \%$ reporta que cuando hay peleas alguno de ellos ha bebido alcohol.

Al indagar por la presencia de problemas debido al consumo de alcohol en algún miembro de la familia, la mayoría (69.5\%) de los estudiantes afirma que sus familiares no han tenido problemas por el consumo excesivo de alcohol; sin embargo, llama la atención que el 30.5\% muestra la existencia de estos problemas en sus familias.

\section{Auto-concepto y Autoestima}

Tabla 5 / Distribución de la población de acuerdo con auto-concepto y autoestima

\begin{tabular}{|c|c|c|c|c|}
\hline & $\begin{array}{l}\text { TOTALMENTE EN } \\
\text { DESACUERDO }\end{array}$ & $\begin{array}{c}\text { EN } \\
\text { DESACUERDO }\end{array}$ & $\begin{array}{c}\text { DE } \\
\text { ACUERDO }\end{array}$ & $\begin{array}{l}\text { TOTALMENTE DE } \\
\text { ACUERDO }\end{array}$ \\
\hline $\begin{array}{l}\text { Siento que tengo } \\
\text { cualidades }\end{array}$ & $6 \%$ & $3 \%$ & $31 \%$ & $60 \%$ \\
\hline $\begin{array}{l}\text { Soy capaz de hacer las } \\
\text { cosas tan bien como casi } \\
\text { toda la gente }\end{array}$ & $7 \%$ & $5 \%$ & $32 \%$ & $55 \%$ \\
\hline $\begin{array}{l}\text { Tengo una actitud positiva } \\
\text { hacia mí mismo/a }\end{array}$ & $7 \%$ & $5 \%$ & $35 \%$ & $54 \%$ \\
\hline $\begin{array}{l}\text { En general estoy } \\
\text { satisfecho/a conmigo } \\
\text { mismo/a }\end{array}$ & $7 \%$ & $6 \%$ & $35 \%$ & $52 \%$ \\
\hline
\end{tabular}


Los resultados exponen que aproximadamente el 93\% tiene una imagen positiva de sí mismo si se contemplan las opciones totalmente de acuerdo y de acuerdo; no obstante, existe aproximadamente un $7 \%$ de estudiantes que tiene una imagen negativa de sí mismo, lo cual los ubica dentro del grupo en situación de vulnerabilidad frente al uso de estas sustancias.

En torno a la percepción del riesgo se mostrarán los resultados relacionados con el consumo de diferentes sustancias.

\section{Percepción del riesgo}

Tabla 6 / Distribución de la población de acuerdo con la percepción de peligrosidad del consumo de drogas lícitas e ilícitas

\begin{tabular}{lccc}
\hline \hline & $\begin{array}{c}\text { NADA } \\
\text { PELIGROSO }\end{array}$ & ES PELIGROSO & $\begin{array}{c}\text { ES MUY } \\
\text { PELIGROSO }\end{array}$ \\
\hline \hline Fumar 1 a 5 cigarrillos diarios & $5 \%$ & $54 \%$ & $41 \%$ \\
Fumar más de 5 cigarros al día & $2 \%$ & $25 \%$ & $73 \%$ \\
Consumir alcohol una vez a la semana & $36 \%$ & $53 \%$ & $11 \%$ \\
Consumir frecuentemente alcohol (más de & $8 \%$ & $47 \%$ & $45 \%$ \\
dos veces a la semana) & $4 \%$ & $36 \%$ & $60 \%$ \\
Consumir drogas médicas sin prescripción & $11 \%$ & $35 \%$ & $54 \%$ \\
Consumir marihuana & $2 \%$ & $21 \%$ & $77 \%$ \\
Consumir cocaína & $2 \%$ & $14 \%$ & $84 \%$ \\
Consumir cocaína en forma de crack, piedra & $2 \%$ & $17 \%$ & $81 \%$ \\
y/o basuco & $2 \%$ & $13 \%$ & $85 \%$ \\
Consumir inhalables & $29 \%$ & $50 \%$ & $21 \%$ \\
Consumir heroína & & & \\
Consumir bebidas energizantes & & &
\end{tabular}

La tabla 6 con respecto al consumo de cigarrillo, muestra que el $41 \%$ de los estudiantes de la Universidad de Boyacá considera que fumar de 1 a 5 cigarrillos diarios es muy peligroso y el $54 \%$ lo considera peligroso. De la misma manera, fumar más de 5 cigarrillos diarios es considerado muy peligroso por el $73 \%$ de los participantes.

$\boldsymbol{E} \| 94$ 
En cuanto al consumo de alcohol, el $53 \%$ de los estudiantes considera peligroso consumir alcohol una vez a la semana, el $36 \%$ lo considera nada peligroso; sin embargo, al preguntar por consumir frecuentemente alcohol, el $92 \%$ lo considera peligroso y muy peligroso.

En lo relacionado con el consumo de medicamentos sin prescripción, el $60 \%$ de los estudiantes lo considera muy peligroso y el $36 \%$, peligroso. Por otro lado el consumo de energizantes es considerado por el $29 \%$ como nada peligroso y por el $50 \%$ como peligroso.

Con respecto a las SPA ilegales, se identifica que el consumo de marihuana es considerado como nada peligroso por el $11 \%$ de los estudiantes. El consumo de otras SPA contempladas en este estudio es percibido como muy peligroso por la mayoría de los estudiantes, los porcentajes se distribuyen de la siguiente manera: Cocaína: 77\%, Bazuco: 84\%, Inhalantes: 81\%, Heroína: 85\%.

Tabla 7 / Distribución de la población de acuerdo con la percepción de consumo de Sustancias Psicoactivas en la Universidad de Boyacá

\begin{tabular}{lcccc}
\hline \hline & NO EXISTE & ES MÍNIMO & $\begin{array}{c}\text { ES } \\
\text { MODERADO }\end{array}$ & ES GRAVE \\
\hline \hline De alcohol: & $4 \%$ & $7 \%$ & $44 \%$ & $46 \%$ \\
De tabaco: & $2 \%$ & $9 \%$ & $40 \%$ & $49 \%$ \\
De drogas (sustancias & $8 \%$ & $36 \%$ & $40 \%$ & $16 \%$ \\
psicoactivas ilegales) & & & & $16 \%$ \\
\hline
\end{tabular}

Con respecto a la percepción de consumo de Sustancias Psicoactivas en la Universidad de Boyacá, la mayoría de los estudiantes considera que el consumo de cigarrillo es grave con un $49 \%$, seguido del consumo de alcohol con un $46 \%$; consideran que el alcohol, tabaco y drogas es moderado. 


\section{Discusión}

El estudio de los factores de riesgo y de protección para el consumo de sustancias ha sido un tema de interés en la investigación de este fenómeno, ya que a partir de su identificación se pueden llegar a establecer hipótesis frente a la probabilidad de consumo en diferentes grupos poblacionales, así como identificar información valiosa para mitigar esta problemática. Como ya se había mencionado anteriormente, estos factores pueden ser ambientales, individuales, genéticos o psicosociales, los cuales pueden modificar el comportamiento frente al consumo de Sustancias Psicoactivas.

Los hallazgos son concordantes con lo expuesto en la literatura identificada frente al tema; con relación al uso del tiempo libre, la mayoría de los estudiantes lo utiliza para hacer tareas o estudiar (63\%) y en segundo lugar para ayudar con las labores de la casa (50\%). Estas situaciones se pueden constituir como un factor protector, pues se deduce que existen normas establecidas dentro de los núcleos familiares que orientan el comportamiento de los estudiantes hacia el cumplimiento de sus responsabilidades (Rivolta, 2012). De la misma manera, si el tiempo dedicado a actividades académicas tiene como resultado el logro de las mismas, se constituye como una situación de protección, ya que los problemas académicos son asociados como predisponentes del consumo de acuerdo con (Lorenzo, Cajaleon \& Gutiérrez, 2012).

Las actividades que desarrollan algunas veces los estudiantes son: Practico algún deporte (61\%), salgo con mi familia (67\%), voy a beber con amigos (59\%), juego videojuegos o veo T.V (58\%), y el $48 \%$ no hace nada especial; practicar algún deporte de acuerdo con la literatura científica se constituye en un factor protector cuando son actividades que alejan a los individuos de entornos sociales y culturales relacionados con el consumo y, disminuyen la ansiedad generada por el consumo (Mosquera \& Galindo, 2010). La misma situación se presenta con el ítem de compartir tiempo con la familia, siempre y cuando no exista un miembro consumidor, esta situación demuestra buenas relaciones familiares, adecuada comunicación y atención de los padres, que es un factor protector de acuerdo con lo encontrado por Medina y Carvalho (2010). Me voy a beber con amigos es otra de las situaciones contempladas, la cual es un factor de riesgo para cualquier SPA ilegal o legal, como tal es un comportamiento de consumo que puede desencadenar el uso de sustancias ilegales. 
Otra de las situaciones relacionadas con el uso del tiempo libre es Jugar video juegos o ver televisión, estas conductas son de los factores de riesgo sociales preponderantes a la hora de determinar el consumo, ya que cuando se muestran personas atractivas y famosas consumiendo alguna sustancia, estos pueden constituirse como modelos para los televidentes, quienes reproducen el comportamiento estudiado (Morales, Plazas, Sánchez, \& Arena, 2011).

En cuanto a la influencia de los pares, se encontró que el 56\% de los amigos de los estudiantes encuestados pertenecen a la universidad. En cuanto al porcentaje de los amigos que fuman resalta el porcentaje de algunos con $57 \%$ y el $21 \%$ de la mayoría, situación que es preocupante ya que se evidencia que en los grupos sociales donde hay consumidores de tabaco y permisividad social se disminuye la percepción negativa del consumo, lo cual se constituye como factor de riesgo (Cogollo, et. al, 2011), ya que puede aumentar la probabilidad de consumo. Por otro lado, el $32 \%$ de los participantes respondieron que la mayoría de sus amigos practican algún deporte y el $48 \%$ tienen buenas calificaciones, situaciones que pueden influir en los participantes como un factor protector. En lo relacionado con los conflictos familiares de los amigos se resalta como aspecto positivo: que solo el $6 \%$ de los participantes reportan la mayoría, el porcentaje restante muestra solo algunos y ninguno, lo cual se constituye como factor protector teniendo en cuenta que un clima familiar negativo, conflictos familiares excesivos, comunicación inadecuada y familias consumidoras, aumentan la probabilidad de consumo (Lorenzo, Cajaleón \& Gutiérrez; 2012).

Contrario a lo anterior, al preguntar a los participantes si sus amigos consumen bebidas alcohólicas, el $42 \%$ reporta que la mayoría y todos usan este tipo de sustancias, situación que es "normalizada" por las culturas debido a la alta permisividad social y la condición de legalidad. Este panorama es preocupante por las consecuencias asociadas al consumo de bebidas embriagantes y por su relación con el inicio de empleo de otras SPA ilegales. Frente al uso de drogas, el $61 \%$ de amigos de los estudiantes que participaron en el estudio no consume drogas, esto muestra un panorama positivo frente a los entornos sociales de los estudiantes.

Con respecto a la presión de grupo, se han identificado estudios que afirman que los estudiantes tienen problemas para decir "no" al momento en que se les ofrece alcohol, lo que lleva a pensar que esta es una variable importante al decidir ingerir este tipo de sustancia (Arias, 
Calderón, Cano, \& Castaño; 2012). Con respecto a esta información, el estudio identificó que el $40 \%$ de los participantes no ha sido presionado para emborracharse y el $31.4 \%$ un poco, en este último porcentaje se ubican aquellas personas que aumentan su probabilidad de consumo y abuso del alcohol.

Aunque la mayoría (69.5\%) de los estudiantes afirma que sus familiares no han tenido problemas por el consumo excesivo de alcohol; sí llama la atención que el 30.5\% muestra la existencia de estos problemas en sus familias, teniendo en cuenta que la literatura científica afirma que familias consumidoras son un factor de riesgo (Lorenzo, Cajaleón \& Gutiérrez; 2012) se debe atender a esta situación para diseñar programas de prevención.

Los resultados exponen que el 93\% tiene una imagen positiva de sí mismo, lo que se insinúa como un factor protector ante el uso de SPA, debido a que estos individuos pueden poseer más mecanismos de defensa para resistir la presión de grupo frente al consumo, así como mayores habilidades sociales. No obstante, existe aproximadamente un $7 \%$ de los estudiantes que tiene una imagen negativa de sí mismos, lo cual los ubica dentro del grupo en situación de vulnerabilidad frente al uso de estas sustancias.

La percepción es considerada desde la psicología como un conjunto de procesos y actividades cognitivas relacionados con la estimulación de los sentidos, mediante las cuales se obtiene información respecto al entorno y a las acciones que se efectúan en él. Se destaca que la percepción influye significativamente en la construcción de prejuicios, estereotipos y que estos pueden influir en la manera en que los individuaos se comportan. Por otro lado, la percepción de riesgo es considerada como el conocimiento de los daños, efectos, grado de susceptibilidad y consecuencias del consumo de drogas (Valdez, 2005).

Con respecto a lo anterior, el $41 \%$ de los estudiantes considera que fumar de 1 a 5 cigarrillo diarios es muy peligroso y el $54 \%$ lo considera peligroso. De la misma manera, fumar más de 5 cigarrillos diarios es considerado muy peligroso por el $73 \%$ de los participantes, esta información coincide con la suministrada por el Ministerio de Protección Social (2014), donde el 88\% de la población que participó en el estudio lo considera de gran riesgo, asimismo, se encontró información similar de acuerdo a los datos suministrados por la Comunidad Andina y la Unión Europea (2009).

$\boldsymbol{E} \| 98$ 
En torno al consumo de alcohol, se entiende que es un comportamiento normal en todas las culturas y ha sido visto a lo largo del tiempo como un elemento propio de la celebración, de acompañamiento y símbolo de reconocimiento social. En el estudio se identificó que el 53\% de los estudiantes considera peligroso consumir esta sustancia una vez a la semana. Sin embargo, el 36\% lo considera nada peligroso, situación que es preocupante ya que esta percepción puede desencadenar patrones de consumo que tengan efectos en la salud física y mental de los estudiantes. El estudio nacional de consumo de Sustancias Psicoactivas realizado en Colombia, muestra que el $88 \%$ de los encuestados considera muy riesgoso consumir alcohol y también identifica que este porcentaje se reduce al 76\% en el grupo de adolescentes (Rivolta, 2012). Nótese que este porcentaje es mucho mayor al identificado en la población estudiantil de la Universidad de Boyacá; sin embargo, al preguntar por consumir frecuentemente alcohol, este porcentaje aumenta y el $92 \%$ los considera peligroso y muy peligroso.

Es importante resaltar que el alcohol y el cigarrillo son SPA legales y existe permisibilidad social ante su uso, situación que podría relacionarse con la prevalencia de consumo; si bien, se considera peligroso, las dinámicas sociales de aceptación reducen la percepción de riesgo frente a su consumo. En lo relacionado con el consumo de medicamentos sin prescripción, el $60 \%$ de los estudiantes lo considera muy peligroso y el $36 \%$, peligroso, estos datos son similares a los reportados por el Ministerio de la Protección Social. Por otro lado, el consumo de energizantes es considerado por el $29 \%$ como nada peligroso y por el $50 \%$ como peligroso.

Respecto a las SPA ilegales, se identifica que el consumo de marihuana es considerado como nada peligroso por el $11 \%$ de los estudiantes, esta información coincide con la encontrada por Jurado (2013), quien afirma que la percepción de riesgo frente a esta sustancia ha venido disminuyendo con el transcurso del tiempo, lo cual está relacionado con el aumento del consumo. El consumo de otras SPA contempladas en este estudio es percibido como muy peligroso por la mayoría de los estudiantes, los porcentajes se distribuyen de la siguiente manera: Cocaína: 77\%, Bazuco: 84\%, Inhalantes: 81\%, Heroína: 85\%. Los porcentajes mostrados anteriormente coinciden con los reportados en el Estudio nacional de consumo de Sustancias Psicoactivas en Colombia 2013, y se resalta que la percepción de peligrosidad disminuye en la población adolescente y la comprendida entre los 18 y 24 años. 
Para finalizar, se puede afirmar que los diferentes factores de riesgo pueden estar presentes o no en un caso determinado, y cuando estos están presentes es más probable que la persona use o abuse de las drogas. Sin embargo, no es garantía para que se presente la conducta de consumo, situación que es similar con los factores de protección. Con relación a la percepción de riesgo, es claro que está influenciada por la legalidad de algunas sustancias y que es relacionada con el uso y abuso de SPA.

\section{Referencias}

Arellanez, J.; Díaz, B.; Wagner, F.; \& Pérez, V. (2004). Factores psicosociales asociados con el abuso y la dependencia de drogas entre adolescentes: Análisis bivariados de un estudio de casos y controles. Salud Mental. 27(3), 54-64. Recuperado de http://www.redalyc.org/articulo.oa?id=58232708

Arias, F.; Calderón, G.; Cano, V.; \& Castaño, G. (2012). Consumo de alcohol y factores de riesgo en estudiantes de dos Universidades Colombianas, AGORA, 12(1), 127-141. Recuperado de: http://web.usbmed.edu.co/ usbmed/elagora/htm/v12nro1/pdf/CONSUMO-ALCOHOL-FACTORES.pdf

Beverido, P., Salas, B., De San Jorge, X., Pavón, P., Blázquez, M., Gogeascoechea, M., \& Cruz, A. (2012). Encuesta sobre consumo de drogas, factores y percepción de riesgo en estudiantes universitarios (CODEU). México: Universidad Veracruzana.

Cáceres, D.; Salazar, I.; Varela, M.; \& Tovar, J. (2006). Consumo de drogas en jóvenes universitarios y su relación de riesgo y protección con los factores psicosociales. Universitas Psychologica, 5(3). 521-534. Recuperado de http://sparta.javeriana.edu.co/psicologia/publicaciones/actualizarrevista/archivos/ V5N306_consumodrogas.pdf

Cid-Monckton, P. \& Pedrao, L. (2011). Factores familiares protectores y de riesgo relacionados al consumo de drogas en adolescentes. Revista Latino-Americana de. Enfermagem, 19, 738-745. Recuperado de http://www.redalyc.org/articulo.oa?id=281421968010

Cogollo, Z.; Arrieta, K.; Blanco, S.; Ramos, L.; Zapata, K.; \& Rodríguez, Y. (2011). Factores psicosociales asociados al consumo de sustancias en estudiantes de una universidad pública. Salud Pública. 13 (3), 470 - 479. Recuperado de http://www.scielosp.org/pdf/rsap/v13n3/v13n3a09.pdf 
Comunidad Andina \& Unión Europea. (2009). Estudio epidemiológico andino sobre consumo de drogas sintéticas en la población universitaria. Informe comparativo: Bolivia, Colombia, Ecuador y Perú. Bogotá: Colombia. Recuperado de: http://www.odc.gov.co/docs/publicaciones_nacionales/Informe\%20Colombia.pdf

García del Castillo, J. (2012). Concepto de percepción de riesgo y su percepción en las adicciones. Health and Addictions / Salud y Drogas. (12) 2, 133-151. Recuperado de http://www.haaj.org/index.php/haaj/ article/download/2/184.

Hernández, R., Fernández, C. \& Baptista, M. (2010). Metodología de la investigación. México: McGraw-Hill.

Lorenzo, M.; Cajaleón, B.; \& Gutiérrez, E. (2012). Prevalencia y factores asociados al consumo de alcohol y tabaco en estudiantes de nutrición de una universidad de Lima- Perú. Revista Peruana de Epidemiología. 16(3) 1-5. Recuperado de http://www.redalyc.org/articulo.oa?id=203125431011

Jurado, L. (2013). Consumo de drogas, percepción de riesgo y adicciones sin sustancias en los jóvenes de la provincia de Córdoba. (Tesis inédita de doctorado). Universidad de Córdoba: España. Recuperada de http:// www.biblioteca.cij.gob.mx/Archivos/Tesis_Digitales/cordoba9.pdf

Manrique, F.; Ospina, J.; \& García, J. (2009). Consumo de alcohol y tabaco en escolares y adolescentes de TunjaColombia. Revista de Salud Pública. 13 (1) 89 - 101. Recuperado de http://www.scielosp.org/pdf/ rsap/v13n1/v13n1a08.pdf

Medina, N. \& Carvalho, M. (2010). Factores protectores de las familias para prevenir el consumo de drogas en un municipio de Colombia. Revista Latino-Americana de Enfermagem, 18 (95), 504-512. Recuperado de http://www.redalyc.org/articulo.oa?id=281421939004 .

Ministerio de la Protección Social \& Instituto Colombiano de Bienestar Familiar. Dirección de Protección. (2010). Lineamiento técnico para el programa especializado de atención a niños, niñas, adolescentes consumidores de sustancias psicoactivas. Bogotá: Colombia.

Ministerio de la Protección Social. (2007). Política nacional para la reducción del consumo de sustancias psicoactivas y su impacto. Bogotá: Colombia. Recuperado de: http://www.descentralizadrogas.gov.co/portals/0/Politica\%0nacional\%20SPA.pdf

Ministerio de la Protección Social. (2014). Estudio Nacional de Consumo de Sustancias Psicoactivas en Colombia. Informe Final. Bogotá: Colombia. Recuperado de: http://www.unodc.org/documents/colombia/2014/ Julio/Estudio_de_Consumo_UNODC.pdf 
Morales, B., Plazas, M., Sánchez, R. \& Arena, C. (2011). Factores de riesgo y de protección relacionados con el consumo de sustancias psicoactivas en estudiantes de enfermería. Latino-Am. Enfermagem. 673 (83), 673-683. Recuperado de http://www.scielo.br/pdf/rlae/v19nspe/03.pdf

Mosquera, J., \& Galindo, J. (2010). Utilización del tiempo libre como factor protector y de riesgo para el consumo de sustancias psicoactivas en jóvenes afrodesplazados de la ciudadela Tokio en Pereira. (Tesis inédita de maestría). Universidad Tecnológica de Pereira, Colombia.

Pastor, G. (2000). Conducta interpersonal: ensayo de Psicología Social Sistémica. Salamanca: Publicaciones Universidad Pontificia.

Peñaloza, I., Pérez, B., Rincón, G. \& Manrique, F. (2010). Consumo de sustancias psicoactivas y factores determinantes en población universitaria de Tunja. Revista de Salud, Historia, Sanidad. 5(2), 1-15. Recuperado de: http://revistas.uptc.edu.co/revistas/index.php/shs/article/view/1888/1880.

Rivolta, S. (2012). Caracterización de los factores familiares de riesgo en el consumo de sustancias, en estudiantes de enseñanza media. Revista Salud Pública. 16(2) 67-81. Recuperado de http://www.saludpublica. fcm.unc.edu.ar/sites/default/files/RSP12_2_10_Caract\%20factores\%20familiares\%20de\%20riesgo\%20en\%20consumo\%20de\%20sustancias.pdf

Valdez, J. (2005). El consumo de alcohol en estudiantes del distrito federal y su relación con la autoestima y la percepción de riesgo. (Tesis inédita de pregrado). Universidad Nacional Autónoma de México, México, D. F.

Villatoro, J. et al. (2009). Encuesta de estudiantes de la Ciudad de México 2006. Prevalencias y evolución del consumo de drogas. Salud Mental, 32(4), 287-297. Recuperado de http://www.scielo.org.mx/pdf/sm/ v32n4/v32n4a4.pdf

Winkelman, M. (2006). Shamanism and the biological origins of religiosity. Shaman. 2(1y2), 89-116. Recuperado de http://www.public.asu.edu/ atmxw/shaman.html

\section{Citación}

Pérez Prada, M.; Barreto Bedoya, P.; Roa Cubaque, M.; Rubiano Díaz, G. (2015). Percepción de riesgo y factores asociados al consumo de drogas legales e ilegales en estudiantes de la Universidad de Boyacá. Enfoques, 1(2).

Recibido: 23 / 02 / 2015 |Aceptado: 10 / 04/ 2015.

E 102 